\title{
An efficient synthesis of 1, 8-dioxo-octahydroxanthenes using tetrabutylammonium hydrogen sulfate
}

\author{
Hitendra N. Karade, Manisha Sathe, and M. P. Kaushik* \\ Process Technology Development Division, Defence $R \&$ \& Establishment, Jhansi Road, \\ Gwalior-474002 (MP), India \\ E-mail: mpkaushik@rediffmail.com
}

\begin{abstract}
A general synthetic route to 1,8-dioxo-octahydroxanthenes has been developed using tetrabutylammonium hydrogen sulfate in semi-aqueous media. The union of two fragments was accomplished by an efficient and convenient protocol enabling the synthesis of 1,8-dioxooctahydroxanthenes in excellent yields. This method provides several advantages such as simple work-up, environmental friendliness and shorter reaction time along with high yields.
\end{abstract}

Keywords: Tetrabutylammonium hydrogen sulfate, 1,8-dioxo-octahydroxanthenes, eco-friendly

\section{Introduction}

Xanthenes are an important class of organic compounds that find use as dyes, fluorescent material for visualization of biomolecules and in laser technologies due to their useful spectroscopic properties. ${ }^{1}$ Xanthenes have also received significant attention from many pharmaceutical and organic chemists essentially because of the broad spectrum of their biological and pharmaceutical properties such as antiviral, ${ }^{2}$ antibacterial, ${ }^{3}$ anti-inflammatory activities $^{4}$ as well as efficiency in photodynamic therapy ${ }^{5}$ and antagonist for the paralyzing action of zoxazolamine. ${ }^{6}$ There are several reports in the literature for the synthesis of 1,8-dioxooctahydroxanthene derivatives employing aromatic aldehydes and 5,5-dimethyl-1,3cyclohexanedione, these include $\mathrm{InCl}_{3} \cdot 4 \mathrm{H}_{2} \mathrm{O}$ in ionic liquid, ${ }^{7}$ solid-state condensation by grinding at room temperature, ${ }^{8} \mathrm{NH}_{2} \mathrm{SO}_{3} \mathrm{H}$ in aqueous media, ${ }^{9} \mathrm{FeCl}_{3} \cdot 6 \mathrm{H}_{2} \mathrm{O}$ in ionic liquid, ${ }^{10}$ microwave-induced synthesis in solid or liquid phase, ${ }^{11} p$-dodecylbenzenesulfonic acid in water. ${ }^{12}$ Each of these methods have their own advantages but also suffer from one or more disadvantages such as prolonged reaction time, tedious work-up processes, low yield, lack of easy availability/preparation of starting materials and hazardous reaction conditions. In addition, chemo selectivity can be a problem, if acid sensitive groups are present in the same molecule. The major disadvantage of some of the methods is that the reaction does not go to completion 
and stops at open chain structure [2,2'-arylmethylene bis(3-hydroxy-5,5-dimethyl-2cyclohexene-1-one) $]^{13}$, instead of forming the cyclized compound. This was overcome by an acid catalyzed cyclization reaction (Scheme 1).

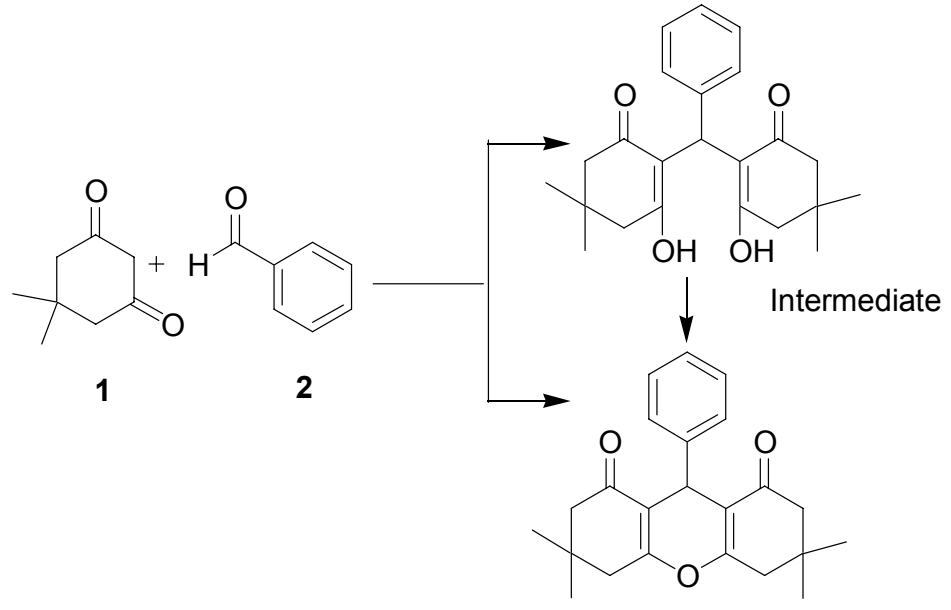

3

Scheme 1. Synthesis of 1,8-dioxo-octahdroxanthene (3) via open chain 2,2'-arylmethylene bis(3hydroxy-5,5-dimethyl-2-cyclohexene-1-one).

Tetrabutylammonium hydrogen sulfate (TBAHS) is an acid catalyst ${ }^{14}$ that has been employed for dehydration and the ring closing step of Hantzsch dihydropyridine like transformations. ${ }^{15}$ This observations inspired us to use the acidic reagent for the synthesis of 1,8 dioxo-octahydroxanthenes. The toxic and volatile nature of many organic solvents, particularly chlorinated hydrocarbons that are widely used in organic synthesis has also posed a serious threat to the environment. Consequently, methods that successfully minimize their use are the focus of much attention. $^{16}$

Herein, we report on the synthesis of 1,8-dioxo-octahydroxanthene using TBAHS in semiaqueous media where 1,4-dioxane acts as co-solvent ( $8 \mathrm{~mL}$ of $\mathrm{H}_{2} \mathrm{O}+2 \mathrm{~mL}$ of 1,4-dioxane) (Scheme 2).

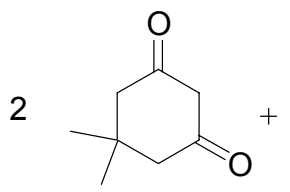

1

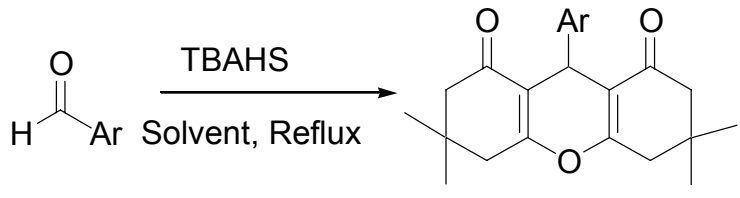

$3 a-3 q$

Scheme 2. Synthesis of 1,8-dioxo-octahydroxanthene using TBAHS in semi-aqueous media. 


\section{Results and Discussion}

Under the given reaction conditions several aromatic aldehydes containing electron donating as well as electron withdrawing groups with diverse substitution pattern were effectively cyclized to give 1,8-dioxo-octahydroxanthenes (Table 1).

Table 1. Synthesis of 9-aryl substituted 1,8-dioxo-octahydroxanthenes using TBAHS

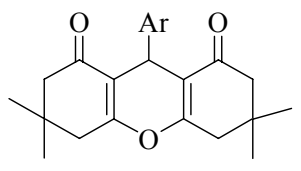

\begin{tabular}{|c|c|c|c|c|c|c|}
\hline \multirow{2}{*}{ Entry } & \multirow{2}{*}{$\mathrm{Ar}$} & \multirow{2}{*}{ Product $^{\mathrm{a}}$} & \multirow{2}{*}{$\begin{array}{l}\text { Time } \\
\text { (hr) }\end{array}$} & \multirow{2}{*}{$\begin{array}{c}\text { Yield } \\
(\%)\end{array}$} & \multicolumn{2}{|c|}{ M.p. ${ }^{\circ} \mathrm{C}$} \\
\hline & & & & & Found & Reported $^{8,12}$ \\
\hline 1. & $\mathrm{C}_{6} \mathrm{H}_{5}$ & $3 a$ & 3.5 & 88 & $204-206$ & $204-205$ \\
\hline 2. & $4-\left(\mathrm{CH}_{3} \mathrm{O}\right)-\mathrm{C}_{6} \mathrm{H}_{4}$ & $3 b$ & 3.0 & 93 & $240-242$ & $241-243$ \\
\hline 3. & 4-( $\left(\mathrm{NMe}_{2}\right)-\mathrm{C}_{6} \mathrm{H}_{4}$ & $3 c$ & 3.5 & 90 & $221-223$ & $220-222$ \\
\hline 4. & $4-(\mathrm{Cl})-\mathrm{C}_{6} \mathrm{H}_{4}$ & 3d & 3.0 & 92 & $229-230$ & $230-231$ \\
\hline 5. & 2-(Cl)- $\mathrm{C}_{6} \mathrm{H}_{4}$ & $3 \mathbf{e}$ & 3.5 & 90 & $225-227$ & $224-226$ \\
\hline 6. & $3-(\mathrm{Cl})-\mathrm{C}_{6} \mathrm{H}_{4}$ & 3f & 3.0 & 94 & $182-184$ & $183-184$ \\
\hline 7. & $2,4-(\mathrm{Cl})_{2}-\mathrm{C}_{6} \mathrm{H}_{3}$ & $3 g$ & 3.0 & 91 & $251-253$ & $253-254$ \\
\hline 8. & $4-\mathrm{NO}_{2}-\mathrm{C}_{6} \mathrm{H}_{4}$ & $3 \mathrm{~h}$ & 3.0 & 94 & $221-223$ & 222 \\
\hline 9. & $3-\mathrm{NO}_{2} \mathrm{C}_{6} \mathrm{H}_{4}$ & $3 \mathbf{i}$ & 3.0 & 94 & $170-172$ & $171.5-172.5$ \\
\hline 10. & $2-\mathrm{NO}_{2} \mathrm{C}_{6} \mathrm{H}_{4}$ & $\mathbf{3 j}$ & 3.5 & 92 & $248-249$ & $246-248$ \\
\hline 11. & $4-\mathrm{HOC}_{6} \mathrm{H}_{4}$ & $3 \mathbf{k}$ & 3.0 & 92 & $247-248$ & $246-247$ \\
\hline 12. & 4- $\mathrm{CH}_{3} \mathrm{C}_{6} \mathrm{H}_{4}$ & 31 & 3.5 & 91 & $217-218$ & $217-218$ \\
\hline 13. & $3,4-\mathrm{OCH}_{2} \mathrm{OC}_{6} \mathrm{H}_{3}$ & $3 \mathbf{m}$ & 3.0 & 92 & $219-220$ & $218.5-220$ \\
\hline 14. & $\mathrm{C}_{6} \mathrm{H}_{5} \mathrm{CH}=\mathrm{CH}$ & $3 \mathbf{n}$ & 3.0 & 90 & $176-78$ & $175-177$ \\
\hline 15. & 3-OEt-4-OH- ${ }_{6} \mathrm{H}_{3}$ & 30 & 3.0 & 92 & $194-96$ & - \\
\hline 16. & $\begin{array}{c}2-\mathrm{OCH}_{3}-5-\mathrm{Br}_{-} \mathrm{C}_{6} \mathrm{H}_{3} \\
\mathrm{O}\end{array}$ & $3 p$ & 3.5 & 90 & 204-06 & - \\
\hline 17. & $\mathrm{H}_{\mathrm{H}}$ & $3 q$ & 3.0 & 90 & $236-238$ & - \\
\hline 18. & 2-Pyridinyl & $3 r$ & 3.5 & 88 & $188-190$ & \\
\hline
\end{tabular}

${ }^{a}$ Experimental data are in supplementary material.

This is a new method for the synthesis of these compounds. By using this method, four new compounds have been synthesized and characterized by all the spectroscopic data. The 
advantage of semi-aqueous solvent system lies in the solubility difference of the starting material and the final product. Hence, during the course of the reaction the product precipitates out and the product was isolated simply by filtration. The practical synthetic efficiency of this reaction was highlighted by the reaction of isophthalaldehyde (4) with dimedone (1) to give a structurally complex xanthenone derivative (3q) (Scheme 3).

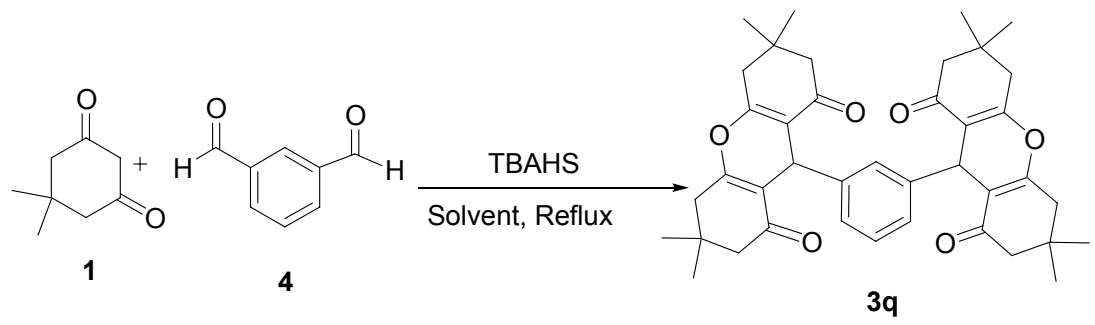

Scheme 3. Synthesis of bis (1,8-dioxo-octahdroxanthene).

An important feature of this method is that the acid sensitive functionality present in the molecule remains unaffected. This fact was amply demonstrated by the reaction of pyridine-2carboxaldehyde (5) with dimedone (1), which gave 9-(pyridin-2-yl)-1,8-dioxooctahydroxanthene (3r) in excellent yield (Scheme 4).

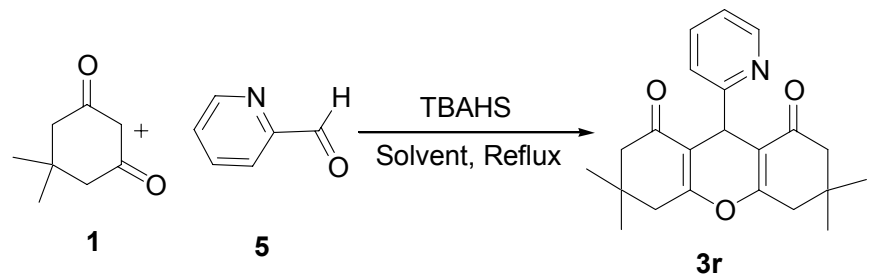

Scheme 4. Synthesis of 9-(pyridin-2-yl)-1,8-dioxo-octahydroxanthene.

\section{Experimental Section}

General experimental procedure. The aldehyde (1 mmol), 5,5-dimethyl-1,3-cyclohexanedione (1) (2 mmol), TBAHS (10 mol \%) were mixed in $20 \mathrm{~mL}$ of aqueous 1,4 -dioxane $(20 \mathrm{~mL}$ of 1,4dioxane $+80 \mathrm{~mL}$ of water) at room temperature. The reaction mixture was refluxed for the time indicated in Table 1. Completion of the reaction was monitored by TLC (Hexane: EtOAc 8: 2). The reaction mixture was cooled to room temperature and the solid product was filtered off and dried. Purity was checked by TLC and all the compounds described gave satisfactory spectroscopic data.

For the typical synthesis of (3q) isopthalaldehyde (4) (1 mmol), dimedone (1) (4 mmol) and TBAHS (10 mol \%) were mixed in the given solvent system and refluxed for $3 \mathrm{hrs}$. After the 
completion of reaction as indicated by TLC, the reaction mixture was cooled to room temperature. Solid product precipitated out and was filtered under suction, washed with cold water $(20 \mathrm{~mL})$ and dried to give the pure product.

3,3,6,6-Tetramethyl-9-(3-ethoxy-4-hydroxyphenyl)-1,8-dioxo-octahydroxanthene (3o). Mp 194-196 ${ }^{\circ} \mathrm{C}$; IR $\left(\mathrm{KBr}, \mathrm{cm}^{-1}\right) v_{\max } 3431,2950,1669,1515,1441,1363,1278,1194 ;{ }^{1} \mathrm{H} \mathrm{NMR}$ $\left(400 \mathrm{MHz}, \mathrm{DMSO}-d_{6}\right) \delta 0.90\left(6 \mathrm{H}, \mathrm{s}, 2 \times \mathrm{CH}_{3}\right), 1.02\left(6 \mathrm{H}, \mathrm{s}, 2 \times \mathrm{CH}_{3}\right), 1.28(3 \mathrm{H}, \mathrm{t}, J=6.9 \mathrm{~Hz}$, $\left.\mathrm{OCH}_{2} \mathrm{CH}_{3}\right), 2.05-2.09$ and 2.23-2.27 (4H, $\left.2 \times \mathrm{d}, J=16.00 \mathrm{~Hz}, 16.00 \mathrm{~Hz}, 2 \times \mathrm{CH}_{2}, \mathrm{H}-4, \mathrm{H}-5\right)$, 2.50-2.53 (4H, d, $\left.12.00 \mathrm{~Hz}, 2 \times \mathrm{CH}_{2}, \mathrm{H}-2, \mathrm{H}-7\right), 3.90\left(2 \mathrm{H}, \mathrm{q}, J=6.9 \mathrm{~Hz},-\mathrm{OCH}_{2} \mathrm{CH}_{3}\right), 4.40(1 \mathrm{H}$, s, H-9), 6.59-6.66 (3H, m, Ar-H), $8.69(1 \mathrm{H}, \mathrm{s},-\mathrm{OH}) ;{ }^{13} \mathrm{C}$ NMR $\left(100 \mathrm{MHz}, \mathrm{DMSO}-d_{6}\right): \delta 14.70$ $\left(\mathrm{CH}_{3},-\mathrm{CH}_{2} \mathrm{CH}_{3}\right), 26.31\left(2 \times \mathrm{CH}_{3}\right), 28.69\left(2 \times \mathrm{CH}_{3}\right), 30.33(\mathrm{C}-3, \mathrm{C}-6), 31.80(\mathrm{C}-9), 38.87(\mathrm{C}-4$, C-5), 50.05 (C-2, C-7), $63.84\left(\mathrm{CH}_{2},-\mathrm{CH}_{2} \mathrm{CH}_{3}\right), 114.00$ (Ar-C), 114.68 (Ar-C), 115.01 (2C, $\mathrm{C}=\mathrm{C}), 120.32$ (Ar-C), 135.28 (Ar-C), $145.22(\mathrm{C}-\mathrm{OH}), 145.87$ (C-OEt), 162.55 (2C, C=C), $196.05(2 \times \mathrm{C}=\mathrm{O})$; ESI-MS m/z: $433(\mathrm{M}+\mathrm{Na}, 5 \%), 273\left(\mathrm{M}^{+}-\mathrm{C}_{8} \mathrm{H}_{9} \mathrm{O}_{2}, 100 \%\right), 843(2 \mathrm{M}+23)$.

3,3,6,6-Tetramethyl-9-(2-methoxy-5-bromophenyl)-1,8-dioxo-octahydroxanthene (3p). Mp 204-206 ${ }^{\circ} \mathrm{C}$; IR $\left(\mathrm{KBr}, \mathrm{cm}^{-1}\right) v_{\max } 2947,2875,1663,1483,1359,1248,1198,883 ;{ }^{1} \mathrm{H}$ NMR (400 $\left.\mathrm{MHz}, \mathrm{DMSO}-d_{6}\right) \delta 0.85\left(6 \mathrm{H}, \mathrm{s}, 2 \times \mathrm{CH}_{3}\right), 1.02\left(6 \mathrm{H}, \mathrm{s}, 2 \times \mathrm{CH}_{3}\right), 1.997-2.037$ and 2.268-2.228 $\left(4 \mathrm{H}, 2 \times \mathrm{d}, J=16.00 \mathrm{~Hz}, 16.00 \mathrm{~Hz}, 2 \times \mathrm{CH}_{2}, \mathrm{H}-4, \mathrm{H}-5\right), 2.54(4 \mathrm{H}, \mathrm{s}, \mathrm{H}-2, \mathrm{H}-7), 3.67$ (s, 3H, OCH3), 4.49 (1H, s, H-9), 6.83-7.31 (3H, m, Ar-H); ${ }^{13} \mathrm{C}$ NMR (100 MHz, DMSO-d 6$): \delta 25.67(2$ $\left.\times \mathrm{CH}_{3}\right), 29.03\left(2 \times \mathrm{CH}_{3}\right), 29.84(2 \mathrm{C}, \mathrm{C}-3, \mathrm{C}-6), 31.90\left(\mathrm{OCH}_{3}\right), 38.87(\mathrm{C}-9), 50.03(2 \mathrm{C}, \mathrm{C}-4, \mathrm{C}-$ 5), 55.52 (2C, C-2, C-7), 111.27 (Ar-C), 111.52 (Ar-C), 113.39 (2C, C=C), 130.25 (Ar-C), 132.29 (Ar-C), 134.10 (Ar-C), 156.94 (Ar-C), 163.91 (2C, C=C), 196.24 (2C, C=O); ESI-MS $m / z: 459(\mathrm{M}+\mathrm{H}, 68 \%), 273\left(\mathrm{M}^{+}-\mathrm{C}_{7} \mathrm{H}_{6} \mathrm{BrO}, 100 \%\right)$.

Bis (1,8-dioxo-octahdroxanthene) (3q). Mp 236-238 ${ }^{\circ} \mathrm{C}$; IR $\left(\mathrm{KBr}, \mathrm{cm}^{-1}\right) v_{\max } 2957,2879,1664$, 1457, 1368, 1203, 1158, 779; ${ }^{1} \mathrm{H}$ NMR (400 MHz, $\left.\mathrm{CDCl}_{3}\right) \delta 1.00\left(12 \mathrm{H}, \mathrm{s}, 4 \times \mathrm{CH}_{3}\right), 1.08(12 \mathrm{H}$, s, $\left.4 \times \mathrm{CH}_{3}\right), 2.10-2.14$ and 2.16-2.20 $\left(8 \mathrm{H}, 2 \times \mathrm{d}, J=16.00 \mathrm{~Hz}, 16.00 \mathrm{~Hz}, 4 \times \mathrm{CH}_{2}\right), 2.398-2.442$ and 2.511-2.555 (8H, $\left.2 \times \mathrm{d}, J=17.6 \mathrm{~Hz}, 17.6 \mathrm{~Hz}, 4 \times \mathrm{CH}_{2}\right), 4.68(2 \mathrm{H}, \mathrm{s}),, 7.04-7.26(4 \mathrm{H}, \mathrm{m}, \mathrm{Ar}-$ $\mathrm{H}) ;{ }^{13} \mathrm{C} \mathrm{NMR}\left(100 \mathrm{MHz}, \mathrm{CDCl}_{3}\right): \delta 27.68\left(4 \times \mathrm{CH}_{3}\right), 29.35\left(4 \times \mathrm{CH}_{3}\right), 31.42(2 \times \mathrm{C}-3,2 \times \mathrm{C}-6)$, $32.32(2 \times \mathrm{C}-9), 40.92(4 \times \mathrm{C}-4, \mathrm{C}-5), 50.91(4 \times \mathrm{C}-2, \mathrm{C}-7), 115.66(4 \times \mathrm{C}=\mathrm{C}), 126.55(\mathrm{Ar}-\mathrm{C})$, 127.95 (Ar-C), 128.39 (Ar-C), $143.74(2 \times$ Ar-C), $162.53(\mathrm{C}=\mathrm{C}), 196.60(4 \times \mathrm{C}=\mathrm{O})$; ESI-MS $m / z: 623(\mathrm{M}+\mathrm{H}, 100 \%), 645(\mathrm{M}+\mathrm{Na}, 40 \%), 661(\mathrm{M}+\mathrm{K}, 20 \%), 273\left(\mathrm{M}^{+}-\mathrm{C}_{23} \mathrm{H}_{25} \mathrm{O}_{3}, 100 \%\right)$.

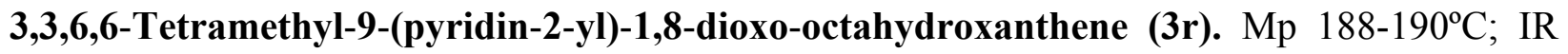
$\left(\mathrm{KBr}, \mathrm{cm}^{-1}\right) v_{\max } 3053,3007,2957,1656,1467,1364,1199,1156,820 ;{ }^{1} \mathrm{H}$ NMR (400 MHz, $\left.\mathrm{CDCl}_{3}\right) \delta 1.00\left(6 \mathrm{H}, \mathrm{s}, 2 \times \mathrm{CH}_{3}\right), 1.10\left(6 \mathrm{H}, \mathrm{s}, 2 \times \mathrm{CH}_{3}\right), 2.13-2.17$ and $2.22-2.26(4 \mathrm{H}, 2 \times \mathrm{d}, J=$ $\left.16.00 \mathrm{~Hz}, 16.00 \mathrm{~Hz}, 2 \times \mathrm{CH}_{2}, \mathrm{H}-4, \mathrm{H}-5\right), 2.435-2.479$ and $2.508-2.552(4 \mathrm{H}, 2 \times \mathrm{d}, J=17.6 \mathrm{~Hz}$, $\left.17.6 \mathrm{~Hz}, 2 \times \mathrm{CH}_{2}, \mathrm{H}-2, \mathrm{H}-7\right), 4.86(1 \mathrm{H}, \mathrm{s}, \mathrm{H}-9), 6.99(1 \mathrm{H}, \mathrm{t}, J=5.3 \mathrm{~Hz}, \mathrm{Ar}-\mathrm{H}), 7.54$ (1H, t, $J=$ $6.1 \mathrm{~Hz}$ Ar-H), $7.60(1 \mathrm{H}, \mathrm{d}, J=7.6 \mathrm{~Hz}), 8.38(1 \mathrm{H}, \mathrm{d}, J=4.52 \mathrm{~Hz}) ;{ }^{13} \mathrm{C} \mathrm{NMR}\left(100 \mathrm{MHz}, \mathrm{CDCl}_{3}\right)$ : $\delta 27.26\left(2 \mathrm{C}, \mathrm{CH}_{3}\right), 29.47\left(2 \mathrm{C}, \mathrm{CH}_{3}\right), 32.42$ (2C C-3, C-6 ), 34.57 (C-9), 41.00 (2C, C-4, C-5), 50.90 (2C, C-2, C-7), 114.47 (2C, C=C), 121.49 (Ar-C), 125.04 (Ar-C), 135.78 (Ar-C), 149.03 
(Ar-C), $161.88(2 \mathrm{C}, \mathrm{C}=\mathrm{C}), 163.47(\mathrm{C}=\mathrm{C}), 197.05$ (2C, C=O); ESI-MS m/z: $352(\mathrm{M}+\mathrm{H}, 100 \%)$, $374(\mathrm{M}+23), 725(2 \mathrm{M}+23)$.

\section{Conclusions}

In summary, we have described a general and efficient procedure for the synthesis of structurally complex and diverse 1,8-dioxo-octahydroxanthenes catalyzed by TBAHS in semi-aqueous media. Moreover, the procedure offers several advantages including high yield, operational simplicity, cleaner reaction and minimal environmental impact, which makes it a useful and attractive process for the synthesis of these compounds.

\section{Acknowledgements}

We thank Shri K. Sekhar, Director DRDE Gwalior for his keen interest and encouragement.

\section{References}

1. (a) Menchen, S. M.; Benson, S. C.; Lam , J. Y. L.; Zhen, W.; Sun, D.; Rosenblum, B. B.; Khan, S. H.; Taing, M. U.S. Patent, US6583168, 2003. (b) Banerjee, A.; Mukherjee, A. K. Stain Technol. 1981, 56, 83.

2. (a) Lambert, R. W.; Martin, J. A.; Merrett, J. H.; Parkes, K. E. B.; Thomas, G. J. PCT Int. Appl. WO 9706178. 1997; Chem. Abstr. 1997, 126, 212377y.

3. Hideo, T. Jpn. Tokkyo Koho JP 56005480, 1981; Chem. Abstr. 1981, 95, 80922b.

4. Poupelin, J. P.; Saint-Rut, G.; Foussard-Blanpin, O.; Narcisse, G.; Uchida- Ernouf, G.; Lacroix, R. Eur. J. Med. Chem. 1978, 13, 67.

5. (a) Ion, R. M. Progr. Catal. 1997, 2, 55; (b) Ion, R. M.; Frackowiak, D.; Planner, A.; Wiktorowicz, K. Acta Biochim. Pol. 1998, 45, 833.

6. (a) Saint-Ruf, G.; De, A.; Hieu, H. T. Bull. Chim. Ther. 1972, 7, 83. (b) Saint-Ruf, G.; Hieu, H. T.; Poupelin, J. P. Naturwiss. 1975, 62, 584.

7. Fan, X.; Hu, X.; Zhang, X.; Wang, J. Can. J. Chem. 2005, 83, 16.

8. Jin, T.-S.; Zhang, J.-S.; Wang, A.-Q.; Li, T.-S. Synth. Commun. 2005, 35, 2339.

9. Jin, T.-S.; Zhang, J.-S.; Wang, A.-Q.; Zhang, F.-S.; Youji Huaxue. 2005, 25, 335: Chem. Abstr. 2005, 143, 4599932.

10. Fan, X. S.; Li, Y. Z.; Zhang, X. Y.; Hu, X. Y.; Wang, J. J. Chin. Chem. Lett. 2005, 16, 897.

11. Tu, S. J.; Gao, Y.; Liu, X. H.; Tang, S. F.; Qiu, X. J. Yoyji Huaxue 2001, 21, 1164 : Chem. Abstr. 2002, 136, 294705x.

12. Jin, T. S.; Zhang, J. S.; Xiao, J. C.; Wang, A. Q.; Li, T. S. Synlett 2004, 866.

13. (a) Horning, E. C.; Horning, M. G.; J. Org. Chem. 1946, 11, 95. (b) Nagarajan, K.; Shenony J. S.; Indian J. Chem. Sec. B 1992, 31, 73. (c) Tu, S. J.; Zhou, J. F.; Lu, Z. S.; Deng, X.; Shi, 
D. Q.; Wang, S. H. Synth. Commun. 2002, 32, 3063. (d) Jin, T. S.; Wang, A. Q.; Ma, H.; Zhang, J. S.; Li, T. S. Indian J. Chem. Sec. B 2006, 45, 470.

14. Otero, M. D.; Batanero, B.; Barba, F. Tetrahedron Lett. 2005, 46, 8681.

15. Tewari, N.; Dwivedi, N.; Tripathi, R. P. Tetrahedron Lett. 2004, 45, 9011.

16. Anastas, P.; Williamson, T. Green Chemistry, Frontiers in Bening Chemical Synthesis and Procedures; Oxford Science Publications: Oxford, 1998. 